\title{
Improvement of the Numerical Method for Effective Refractive Index Calculation of Porous Composite Materials Using Microlevel Models
}

\author{
N. AndrushchaK*, N. JaWorski and M. Lobur \\ CAD Department, Lviv Polytechnic National University, 12 S. Bandery Str., Lviv, Ukraine \\ (Received June 26, 2017; in final form October 20, 2017)
}

\begin{abstract}
The numerical method of the effective refractive index of porous composite materials calculation was improved by implementation of microlevel cellular structural models. The proposed solution gives the ability uniformly to analyse complex structural inhomogeneities and to synthesize the corresponding index based on the simulation of electrostatic field. The proposed realization is simpler and takes less computations capacities in comparison to analogous simulation methods. The method was probed on the example of $\mathrm{SiO}_{2}$ nanoporous matrix with refractive index $n=1.426$ at $\lambda=2.71 \mu \mathrm{m}$ wavelength. The results of simulation are in good agreement with other analytical models from literature.
\end{abstract}

DOI: 10.12693/APhysPolA.133.164

PACS/topics: 02.70.Dh, 41.20.Cv, 78.20.Ci, 81.05.Rm

\section{Introduction}

Porous composite materials, such as anodic alumina $\left(\mathrm{Al}_{2} \mathrm{O}_{3}\right)$, silicon dioxide $\left(\mathrm{SiO}_{2}\right)$, titanium dioxide $\left(\mathrm{TiO}_{2}\right)$ and others are commonly used for development of microsystems, environment sensors, filtering elements for micro- and nanometric filtration and substances separation, matrices for nanoparticles synthesis, nanoelectronic devices, etc. Such variety of applications is possible because of the available porous phase with self-organized, usually hexagonal, pores placement [1-4].

The actual task of porous materials investigation is in finding the effective physical characteristics of those porous composites, in particular the effective refractive index, which depends on structural parameters of the composite. Previously we have developed setups for experimental testings of the crystalline materials in wide frequency range [5-11]. However, with development of new materials new methods for rapid composite materials evaluation are crucial. Investigation of these new methods become even more necessary if pores of the composite are filled with another crystalline material [12].

In addition to the experimental verification of the results, the numerical simulation of the composites is a powerful tool for receiving results on the investigated structure. Furthermore, the numerical methods and models that describe corresponding physical process within composite structure are used to achieve results with the maximal accuracy [13]. Typically, they are based on the finite element method [14]. Thus, using the combinations of different methods and approaches in the present paper the numerical method for the effective refractive index calculation of the porous crystalline

*corresponding author; e-mail: nandrush@gmail.com composites is adapted and improved. Due to the usage of microlevel cell models, the proposed method gives the ability to analyze the impact of material complex structural inhomogeneities

\section{Calculation of the effective characteristics of composite materials}

Each composite has some specific set of characteristics, which depend on the structural parameters. The calculation of characteristics dependences can be done by homogenization process. There are three main model classes for this purpose [13-15]: (1) empirical model that is based on the interpolation between natural experiment results, (2) simple structural model that is based on known analytical models of effective fields or on numerical averaging models that describe local interconnection between composite matrix and one of its components, (3) complex microlevel model that is based on numerical methods and allow one to analyze composite components inhomogeneities.

Analytical Drude model can be used for composites with porous structure $[2,3,16]$. Out of the differential equations of the Drude model one can get the following equation:

$$
n_{\text {eff }}^{2}=(1-P) n_{m}^{2}+P n_{p}^{2},
$$

where $P$ is a porosity, $n_{m}$ is the characteristic of composite matrix, $n_{p}$ is the characteristic of porous phase. Equation (1) is the particular case of the Bragg and Pipard model [16] for the case of ideal cylindrical pores. It can be used for calculation of all linear conductance characteristics with different physical nature, but only when physical flux is along the direction of the pores orientation. The Drude model describes the upper boundary for refractive coefficient. The lower boundary describes the known model of a parallel connection of conductivities, from which the effective refractive index can be expressed 


$$
n_{\text {eff }}=(1-P) n_{m}+P n_{p} .
$$

The analysis and comparative characteristics of those and set of other models are presented in different literature references, in particular in [17].

As it is known, microlevel model of effective refractive index calculation is the model that is based on transverse electromagnetic waves propagation in composite materials by finite element method of numerical simulation $[2,3]$. In this paper the model of electrostatic field simulation is used for crystalline composites.

\section{Generation of the structural model of porous composite}

Typically, the simulation of porous composite structure is related to pores formation process [1]. However, it is a separate complex task and featured characteristics of ending structure can be described by simpler methods, such as simulation of cylindrical or fibrous inclusions.

The cellular models are used in this paper for generation of porous composites structural models [18, 19]. These models are used in the form of elementary volumes of composite materials that contain big amount of regular voxel cells and simultaneously can be used as a finite element discretization. Applying such formalization, the structural model is described as a matrix of scalar intensities in the range from 0 to 1 . Sub-ranges of these intensities determine the components of composite materials. The Bezier curves are used to generate random fibrous inclusions that describe composite material pores. The generation method is described in [4, 18]. Each pore is placed in the basic coordinate of the elemental volume, which is a node of hexagonal tessellation. Applying the cross sections of the intensities of elementary cells at a certain level allows to define subranges and thus to control the size of the pores. The usage of the Bezier curves enables the pores to be modeled taking into account nanostructural heterogeneities observed in ordered and disordered areas of real porous composites. Moreover, the further usage of the method of generating random transition layers [20] makes it possible to investigate structural heterogeneities of pore sides. The resulting structural model can be directly used as finite-element sampling by combining each of the eight adjacent cells into a cube with its subsequent partition into six simplexes.

\section{Effective refraction index calculation}

Let us consider dielectrics such as porous composites that are used in optics. It can be assumed that there are no magnetic field applied and the wavelength of electromagnetic field is constant $(\lambda=$ const). In that case, from the known system of the Maxwell equations one can derive an elliptic equation for the potential of the electrostatic field

$$
\varepsilon \nabla^{2} U=0 \text {, }
$$

where $\varepsilon$ is a permittivity, $U$ is a potential. Further, let us consider a model of homogeneous elemental volume of composite material in the form of a cube [14, 21], then permittivity can be found as

$$
\varepsilon=\frac{d \cdot \rho}{\Gamma_{\rho}\left(U_{\Gamma_{\rho}}-U_{\infty}\right)},
$$

where $\Gamma_{\rho}$ is the surface area of the elemental volume $\Omega$, and the flow density of the charge is given as $\rho$, and $U_{\infty}$ is a potential on the opposite side, $d$ is a length of the elemental volume. The boundary value problem can be defined as:

$$
\varepsilon \nabla^{2} U(\boldsymbol{r})=0, \boldsymbol{r} \in \Omega,\left.\quad \frac{\partial U}{\partial \boldsymbol{n}}\right|_{\Gamma_{q}} \rho, \boldsymbol{n} \perp \Gamma_{\rho},\left.U\right|_{\Gamma_{U}}=U_{\infty} .
$$

The solution to the (5) problem by finite element method was proposed by authors in [14] for the case of stationary heat conduction and elasticity linear problems modeling in terms of combined formalization of coupled thermoelectricity problems simulation in complex structured composite materials.

The elements at boundaries can be considered as the ends of separated conductors that are connected in parallel (in relation to the density of charge flux). Thus, in the current paper, by applying the electrostatic analogy method [14, 21], the discrete model, that is built on composite material microlevel structural model discretization by finite simplex elements, leads to equation [21] for effective permittivity calculation. As it is known, for dielectrics $n^{2}=\varepsilon$, thus the effective refractive index of the investigated composite material can be expressed as

$$
\begin{gathered}
n_{e f f}^{2}=d \rho / \iint_{\Gamma_{\rho}}\left(U_{\Gamma_{\rho}}-U_{\infty}\right) \mathrm{d} \Gamma_{\rho}= \\
\frac{d \rho}{\Gamma_{\rho}} \sum_{j=1}^{P_{\Gamma_{\rho}}} 3\left(\Gamma_{\rho}\right)_{j} / \sum_{i=1}^{3}\left(\left(U_{\rho}\right)_{i, j}-U_{\infty}\right),
\end{gathered}
$$

where 3 is a number of surface finite element nodes, $\left(U_{\Gamma_{\rho}}\right)_{i, j}$ is a potential at $i$-th node of $j$-th surface finite element, $P_{\Gamma_{\rho}}$ is a number of surface finite elements.

\section{Results of simulation}

The improved method was implemented by $\mathrm{C}++11$ algorithmic language using OpenCL v.1.2 technology (GPU Cedar, AMD Radeon HD 6300M Series) and Qt SDK v.5.4.1. As the operating system Windows 7 Ultimate x64 was used. The program was linked under x64 by MinGW 4.9.2 compiler. Simulations were conducted on a PC with average configuration. The results are shown in Fig. 1 and Fig. 2.

As it can be seen from Fig. 2, at zero deviation, the results are consistent with the Drude model within the limits of the computational error, since the pores are described by ideal cylindrical inclusions.

\section{Conclusions}

In this paper, the method of effective refractive index calculation of porous composite materials was improved by using the microlevel cellular structural model, the method of random fibrous inclusions generation using the Bezier curves, the numerical finite element model of electrostatic processes, and the method of electrostatic 

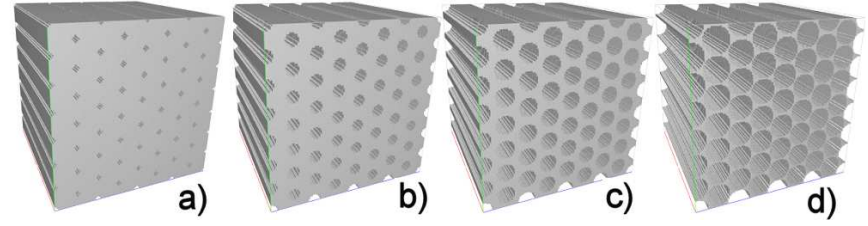

Fig. 1. The results of porous composite material structure modeling: elemental volume with $128^{3}$ cells in equivalent to $84 \mathrm{~nm}^{3}$; matrix $\mathrm{SiO}_{2}$ with refractive index $n=1.426($ at $\lambda=2.71 \mu \mathrm{m})[3]$; phase of reinforcement with refractive index $n=1$ - porous cylindrical inclusions that are approximated by first order Bezier curves with interpore distance of $12 \mathrm{~nm}$, maximum pore diameter $D_{p}=15 \mathrm{~nm}$; the change in porosity $P$ occurred due to the application of the section of cells intensities, which is equivalent to changing the diameter of the pore $D_{p}$ : a) $-P=4.44 \%, D_{p}=3 \mathrm{~nm}$; b) $-P=20.85 \%$, $\left.\left.D_{p}=6 \mathrm{~nm} ; \mathrm{c}\right)-P=46.83 \%, D_{p}=9 \mathrm{~nm} ; \mathrm{d}\right)-$ $P=82.37 \%, D_{p}=12 \mathrm{~nm}$.
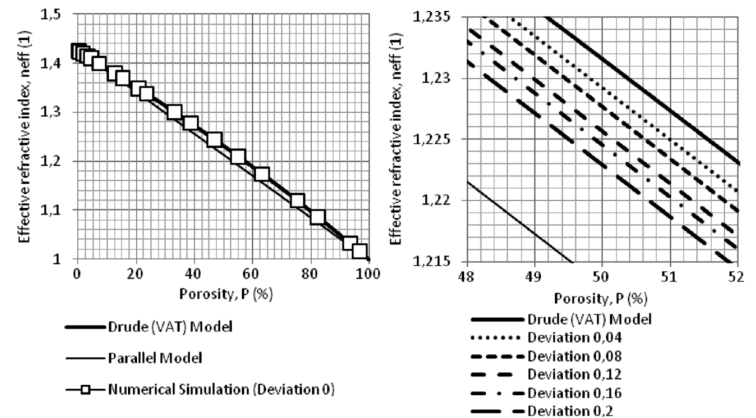

Fig. 2. Calculated effective refractive index of porous composite material with different deviations of the pores from linear orientation - the pores are approximated by 5th order Bezier curves by 10 linear segments per curve and each curve node is uniformly deviated from linear direction in the range from 0 to 0.2 from the length of the elemental volume.

analogies. The proposed method of effective refractive index calculation of porous composite materials, in comparison to the analytical methods, allows consideration of complex structural heterogeneities of the material, and directly apply it as a regular discretization and thereby reduce the number of necessary calculations. Therefore, such approach of effective refraction index modeling of the porous composite based on the results of numerical simulation of the electrostatic field is simpler to implement and requires less computing powers in compare to similar numerical methods. The method was tested and proved its feasibility on the example of $\mathrm{SiO}_{2}$ nanoporous matrix with refractive index $n=1.426$ at $\lambda=2.71 \mu \mathrm{m}$ wavelength. The results of simulation are in good agreement with other analytical models from literature.

\section{References}

[1] A. Losic, Nanoporous Alumina. Fabrication, Structure, Properties and Applications, Springer, New York 2015 , p. 371.

[2] N. Hutchinson, et al., Thin Solid Films 518, 2141 (2010).
[3] M. Braun, L. Pilon, Thin Solid Films 496, 505 (2006).

[4] N.B. Jaworski, N.A. Andrushchak, in: Proc. 14 th Int. Conf. The Experience of Designing and Application of CAD Systems in Microelectronics, CADSM 2017, Polyana-Svalyava (Ukraine) 2017, p. 63.

[5] N. Andrushchak, in: Proc. 13th Int. Conf. on Modern Problems of Radio Engineering, Telecommunications and Computer Science, Slavsko (Ukraine) 2016, p. 186.

[6] N.A. Andrushchak, I.D. Karbovnyk, K. Godziszewski, Ye. Yashchyshyn, M.V. Lobur, A.S. Andrushchak, IEEE Trans. Instrum. Meas. 64, 3005 (2015).

[7] N.A. Andrushchak, O.I. Syrotynsky, I.D. Karbovnyk, Ya.V. Bobitskii, A.S. Andrushchak, A.V. Kityk, Microwave Opt. Technol. Lett. 53, 1193 (2011).

[8] N.A. Andrushchak, Ya.V. Bobitskii, T.V. Maksymyuk, O.I. Syrotynsky, A.S. Andrushchak, I.D. Karbovnyk, in: Proc. 4th Microwave and Radar Week, 18th Intern. Conf. on Microwaves, Radar and Wireless Conmunications, MIKON 2010, Lithuania 2010, p. 273.

[9] I.D. Karbovnyk, N.A. Andrushchak, Ya.V. Bobitskii, in: Proc. 5th Int. Conf. on Advanced Optoelectronics and Lasers, CAOL'2010, Sevastopol (Ukraine) 2010, p. 226.

[10] A.S. Andrushchak, O.I. Syrotynskiy, N.A. Andrushchak, in: Proc. Int. Workshop - THz Radiation: Basic Research and Applications, TERA, 2008, p. 174 .

[11] A.S. Andrushchak, T.I. Voronyak, O.V. Yurkevych, N.A. Andrushchak, A.V. Kityk, Opt. Lasers Eng. 51, $342(2013)$.

[12] N.A. Andrushchak, O.A. Buryy, V.T. Adamiv, I.M. Teslyuk, A.S. Andrushchak, A.V. Kityk, in: Proc. 6th Int. Conf. Nanomaterials: Applications and Properties, NAP 2016, Lviv (Ukraine) 2016, p. 02NNSA10-1.

[13] N. Jaworski, et al., in: Proc. 8th Int. Scientific and Technical Conf. CSIT'2013, Lviv (Ukraine) 2013, p. 46.

[14] N. Jaworski, Econtechmod 4, 2 (2015).

[15] V. Narusberh, G. Teters, Stability and Optimization of Composite Shells, Zynatne, Riga 1988 (in Russian).

[16] N. Sahoo, et al., Appl. Surf. Sci. 253, 6787 (2007).

[17] M. Wang, N. Pan, Int. J. Mater. Sci. Eng. 63, 1 (2008).

[18] N. Jaworski, et al., Sci. Bull. Ukr. Nation. Forestry Univ. 25, 359 (2015).

[19] S. Torquato, Random Heterogeneous Materials. Microstructure and Macroscopic Properties, Springer, New York 2002.

[20] N. Jaworski, et al., Bull. LPNU Comp. Sci. Inf. Tech. 826, 385 (2015).

[21] N. Jaworski, et al., Bull. LPNU Comp. Sys. Des. 747, 59 (2012). 